\title{
Fuzzy AHP and TOPSIS in Cross Domain Collaboration Recommendation with Fuzzy Visualization Representation
}

\author{
Maslina Zolkepli and Teh Noranis Mohd. Aris
}

\begin{abstract}
Cross domain collaboration recommendation method is proposed by combining fuzzy Analytic Hierarchy Process (AHP), fuzzy Technique for Order Preference by Similarity to Ideal Solution (TOPSIS), and fuzzy network graph for interactive visualization method. Existing cross-domain recommendation tackles the problem of sparsity, scalability, cold-start and serendipity issues found in single-domain, therefore the combination of fuzzy AHP and TOPSIS with visualization method may be able to give decision makers a quick start to initiate cross-domain collaborations. The proposed method is applied to the DBLP bibliographic citation dataset that consists of $\mathbf{1 0}$ domains in the field of computer science. Results show that the combination of fuzzy AHP and TOPSIS enables decision makers to find several authors from across domains that consist of $\mathbf{2 . 2}$ million publications in less than 3 minutes. The combination method will be represented in fuzzy visualization technique for fuzzy data. The establishment of the cross domain recommendation will set a stage for efficient preparation for researchers who are interested to venture into other domains to increase their research competency.
\end{abstract}

Index Terms-Cross domain recommendation, fuzzy AHP, fuzzy TOPSIS, fuzzy visualization, recommendation system.

\section{INTRODUCTION}

Cross domain collaborations has garnered much interest from researchers in the field of data mining and knowledge discovery in the recent years. While the research in this area is flourishing, there is still very limited success in terms of realization for general research use. Up until today, specifically in UPM, cross domain collaboration are still initiated through word of mouth. Therefore, a tool that can recommend possible collaboration among diversified fields is very much beneficial.

There is increasing need for researchers to do cross domain collaboration as it allows researchers to enter a new field of research, keep their research skills up to date and also the opportunity to learn other domain research language, growing speed and rewards. Given the lack of cross domain collaboration recommendation tool available out in the market, a cross domain recommendation framework represented in fuzzy visualization is proposed.

The proposed method will be beneficial to improve the task of finding the connection among different domains

Manuscript received July 14, 2019; revised October 17, 2019. This work was supported by the Universiti Putra Malaysia under Geran Putra (GP/2017/9569500).

The authors are with the Dept. of Computer Science, Faculty of Computer Science and Information Technology, Universiti Putra Malaysia, 43400 UPM Serdang, Selangor, Malaysia (e-mail: masz@upm.edu.my, nuranis@upm.edu.my). quickly and efficiently. Cross domain collaboration recommendation systems have attracted attention to help researchers searching for possible collaborations among different fields. Current recommendation require analyzing each topics in desired domain and collecting information from many sources such as social circles and related research lab websites. There are several cross domain collaboration recommendation approaches that have been proposed in recent years, such as the cross domain topic learning (CTL) [1] that handles sparse connection, complementary exercise and topic skewness. A framework has been developed for folksonomies cross domain recommendation [2] that creates tags of users' profile based on the relationship of tags among multiple domains. Content-Based Cross-Domain Recommendations framework using segmented models [3] can be implemented with various classifiers and transfer common information among different domains. Cross domain recommendation using multidimensional tensor factorization [4] trades off influence among domains optimally. It compares the sparsity, scalability, cold-start and serendipity issues found in single-domain recommendation system. Another cross domain recommender system [5] transfers knowledge from the source rating matrix to help increase the prediction accuracy of the recommender system on the target rating matrix to ensure consistent information transfer between domains.

While these existing approaches gives recommendation for cross domain collaboration, they do not offer details on how much each recommended domain relates to the other domain. In this proposal, we aimed to develop a cross domain collaboration recommendation framework to solve multi-criteria decision making (MCDM) problems based on fuzzy analytic hierarchy process (fuzzy AHP) [6] and fuzzy Technique for Order Performance by Similarity to Ideal Solution (fuzzy TOPSIS) [7] methods. In order to evaluate the imprecise judgements of decision makers when deciding which domain to collaborate with, a fuzzy AHP and fuzzy TOPSIS decision making framework is proposed as an evaluation tool to make the cross domain recommendations. Unlike the conventional combination of fuzzy AHP and fuzzy TOPSIS [8] methods, the proposed method is represented with fuzzy visualization [9]-[13] that is able to convey degree of precision of each recommendation given. This will help shorten the time taken for decision makers to understand the structure and characteristic of the recommendation information.

The rest of the paper is organized as follows: Section II describes the fuzzy AHP, fuzzy TOPSIS and the integration of fuzzy AHP and fuzzy TOPSIS. In Section III the DBLP citation big data with ten domains in computer science is explained. Section IV describes the fuzzy visualization to 
present the recommendation result. In Section $\mathrm{V}$ the experiment result is explained by applying the integration of fuzzy AHP and fuzzy TOPSIS algorithms on the DBLP citation big data.

\section{COMBINATION OF FUZZY AHP AND FUZZY TOPSIS METHOD FOR CROSS-DOMAIN COLLABORATION RECOMMENDATION}

Many methods are suggested for cross-domain recommendation. In the proposed framework, fuzzy AHP and fuzzy TOPSIS methods are chosen to produce the cross domain collaboration recommendation.

\section{A. Fuzzy Set Theory}

Fuzzy set theory [14] targeted to model the vagueness or imprecision of human cognitive processes. The main idea of fuzzy set theory is that an element has a degree of membership in a fuzzy set [15], [16]. A fuzzy set is defined by a membership function that maps elements to degrees of membership within a certain interval, which is usually $[0,1]$. If the membership degree is zero, the element does not belong to the set. If the membership degree is one, the element belongs completely to the set. Finally, if the value lies within the interval of one and zero, the element has a certain degree of membership, which means that it partially belong to the set [17].

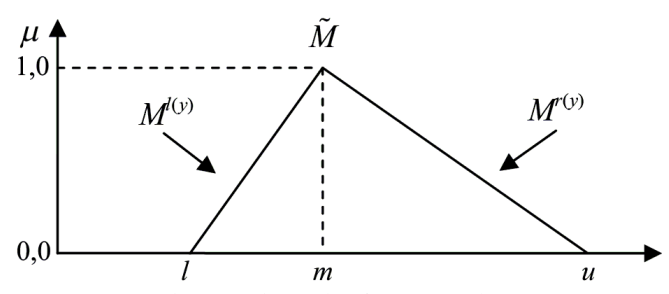

Fig. 1. Triangular fuzzy number.

A Triangular Fuzzy Number (TFN) $\tilde{M}$ is shown in Fig. 1 . A TFN is denoted as $(a, b, c)$. The parameters $a, b$ and $c(a \leq b$ $\leq c$ ), denote the smallest possible value, the most promising value, and the largest possible value that describe a fuzzy event.

Each TFN has linear representations on its left and right side, such that its membership function can be defined as:

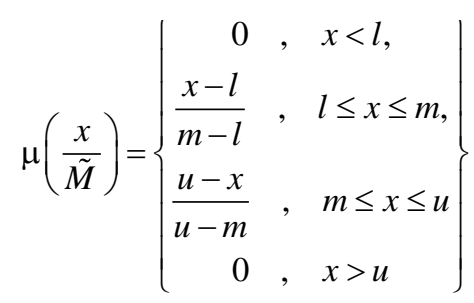

A fuzzy number (FN) can always be given by its corresponding left and right representation of each degree of membership as follows:

$$
\tilde{M}=M^{l(y)}, M^{r(y)}=[l+(m-l) y, u+(m-u) y], y \in[0,1] .
$$

where $I(y)$ and $r(y)$ denote the left side representation and the right side representation of a fuzzy number (FN), respectively. There are a number of ranking methods [18] have been introduced that may provide different ranking results.

\section{B. Fuzzy AHP}

The AHP [19] is a quantitative technique that structures a multi-attribute, multi-person and multi-period problem hierarchically so that solutions are facilitated. The main advantage of AHP is that it is effective in handling multiple criteria in order to make decisions and it is able to cater to qualitative and quantitative data [20]. It accepts the pair-wise comparison of each alternative that belong to each criterion and offers a decision support tool for multi-criteria decision making problems. In classic AHP method, the objective is situated in the first level, the criteria is situated in the second level, and the available alternatives is situated in the third level [21]. Classic AHP does not take the vagueness of the criteria into consideration when making decision. Therefore, fuzzy AHP was proposed to equip classic AHP with the ability to accept uncertainty and ambiguity in human thinking style [22].

Fuzzy AHP is a technique where the pair wise comparisons of the criteria and the alternatives are achieved by using fuzzy triangular numbers to represent linguistic variables [23]. One of the earliest application of fuzzy AHP provides more realistic results than the original crisp method [24] where the choice is between a number of alternatives under overlapping judgements. Fuzzy numbers was used to represent expert opinion in ranking alternatives across a set of criteria [25]. The fuzzy numbers were used to describe experts' preferences and fuzzy arithmetic was used to compute fuzzy ranking. There are many more fuzzy AHP related techniques that are available, but the Buckley method was selected for this study to determine the fuzzy weights of importance for the criteria and the alternatives.

\section{Fuzzy TOPSIS}

Technique for order preference by similarity to an ideal solution (TOPSIS) is another classical multiple criteria decision making (MCDM) [26] method. In TOPSIS, the chosen alternative should have the shortest distance from the positive ideal solution and the farthest distance from the negative ideal solution. The two reference points, shortest point and farthest points do not take the relative importance of their distance from one another into consideration. As there exist have various types of vagueness and imprecision in assessment values, crisp decision-making technique is not able to handle these decision making problems. Therefore, a fuzzy extension of TOPSIS method is needed. A number of fuzzy TOPSIS methods and applications have been developed in recent years [27]-[29].

In fuzzy TOPSIS, evaluations are expressed in linguistic terms and mapped into fuzzy numbers. It requires preliminary information regarding the weightage of the criteria. The weight is expressed by assigning a value to each considered criterion. The value of each criterion is evaluated by fuzzy AHP [30].

\section{Combination of Fuzzy AHP and Fuzzy TOPSIS}

A combination of fuzzy AHP and fuzzy TOPSIS is proposed, where fuzzy AHP approach will be conducted first, before utilizing the steps of fuzzy TOPSIS. Fig. 2 shows the cross domain recommendation framework with fuzzy AHP 
and fuzzy TOPSIS methods. The steps of the framework are as follows:

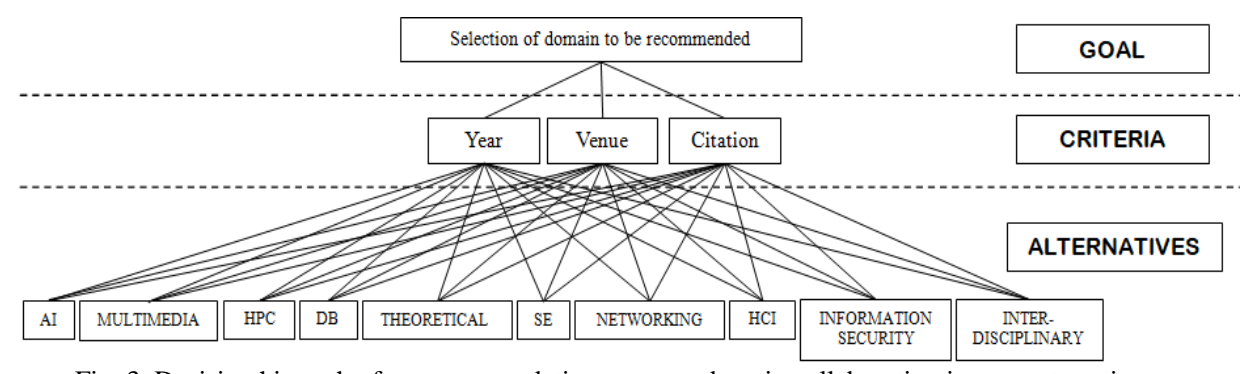

Fig. 3. Decision hierarchy for recommendation on cross domain collaboration in computer science.

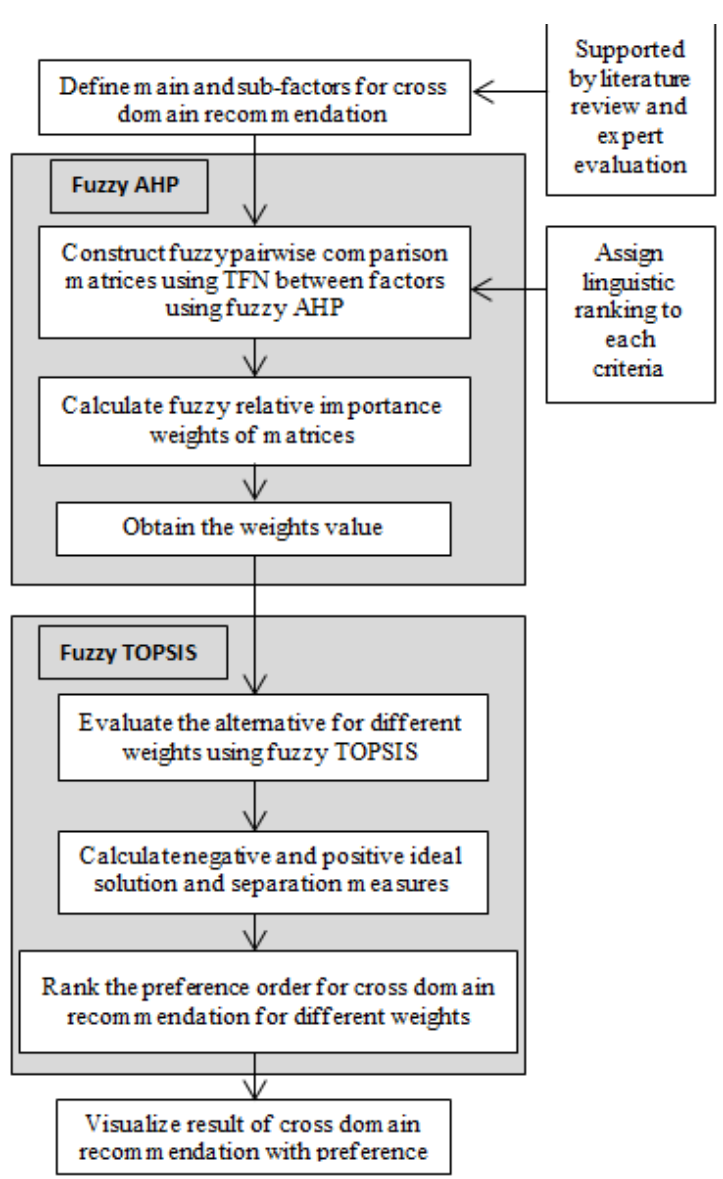

Fig. 2. Fuzzy AHP and Fuzzy TOPSIS analysis framework for cross domain recommendation. DBLP bibliographic citation big data with 10 domains.

\section{1) Part I - Cross domain recommendation evaluation criteria}

In cross domain recommendation, the objective is to find out the best domain that matched the keywords entered by users. Fig. 3 depicts the decision hierarchy for cross domain recommendation.

2) Part II - Determining cross domain recommendation evaluation criteria weights

Firstly, pair wise comparisons are performed in linguistic terms. Then the required data for the analysis are entered and the fuzzy comparison matrices are calculated. Once the weights' values have been obtained, it will become the input for the fuzzy TOPSIS method.

3) Part III - Ranking the cross domain recommendation

Evaluate the alternatives for different weights. Next, calculate negative and positive ideal solution and separation measures.

Lastly, rank the preference order for cross domain recommendation for different weights.
4) Part IV - Visualization of the cross domain recommendation ranking to the users

Through the visualization system, the result of cross domain recommendation with preference order will be shown to users, in different colors to set the difference.

The dataset used to test the method is the DBLP and ACM citation data that are categorized into 10 domains in computer science. The domains are 'Artificial Intelligence', 'Computer Graphics and Multimedia', 'Computer Networks', 'Database, Data Mining and Information Retrieval', 'High Performance Computing', 'Human Computer Interaction and Ubiquitous Computing', 'Information Security', 'Interdisciplinary Studies', 'Software Engineering', and 'Theoretical Computer Science'.

Recommendation is discovered through publications published in each domain. By evaluating the publication title, year, venue, and user preference, fuzzy AHP and TOPSIS is used to select the best recommendation for the researcher. For example, a researcher is looking for a domain to collaborate with. There are 10 domains that can be recommended to the researcher. Each domain has four attributes that can be specified by the researcher. They are researchers' keyword, year of publication, venue of publication and the title of the publication. In order to evaluate the domains to be recommended, three criteria has been selected for the performance assessment. The criteria are shown in Table I.

\begin{tabular}{lll}
\multicolumn{3}{l}{ TABLE I: ASSESSMENT CRITERIA FOR CROSS DOMAIN RECOMMENDATION } \\
\hline \hline Criteria & Description & Value Range \\
\hline Year & $\begin{array}{l}\text { The year the research paper is } \\
\text { published. }\end{array}$ & $1994-2014$ \\
SJR & $\begin{array}{l}\text { SCImago Journal Rank value of the } \\
\text { publication venue. }\end{array}$ & $0.001-4.000$ \\
Citation & Total citation of the research paper. & $0-30$ \\
\hline \hline
\end{tabular}

\section{EXPERIMENTS ON PUBLICATIONS FROM 10 DOMAINS IN COMPUTER SCIENCE}

For 'Citation' criterion, the citation is considered as 'Very Few' if the citation count is 10 or below, 'Few' is the citation count is between 5 and 25, and 'Many' if the citation count is 20 or more.

Fig. 4 shows the membership function for 'Citation' criterion. For 'Year' criterion, the publication is considered 'Old' if it is published in the last 10 to 30 years, 'Recent' if it is published between 5 to 15 years ago, and 'Most Recent' if it is published within the last 5 years. Fig. 5 shows the membership function for 'Year' criterion. For 'Scientific Journal Ranking' criterion, the SJR of the journal is 
considered 'Low' if the score is between 0.000 and 1.800, 'Medium' if the score is between 1.000 and 3.000, and 'High' if the score is higher than 2.300. Fig. 6 shows the membership function for 'SJR' criterion.

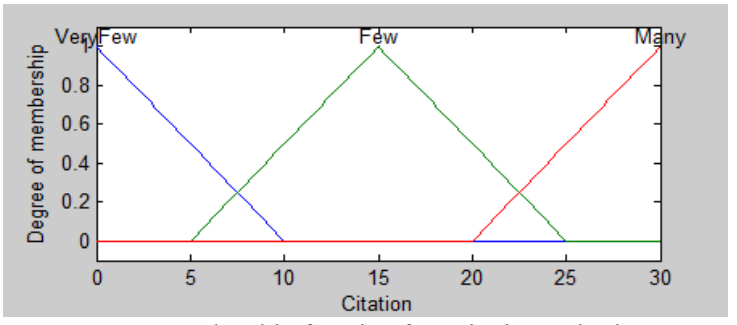

Fig. 4. Membership function for 'Citation' criterion.

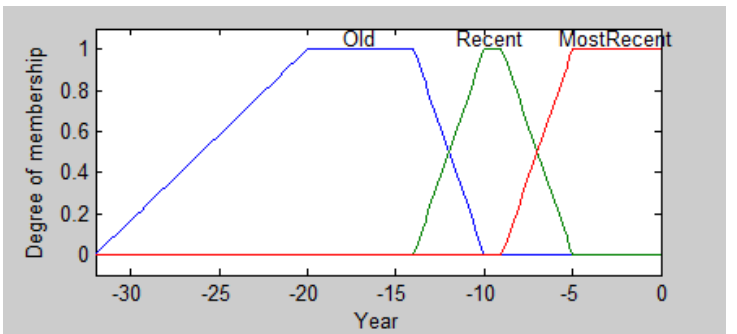

Fig. 5. Membership function for 'Year' criterion.

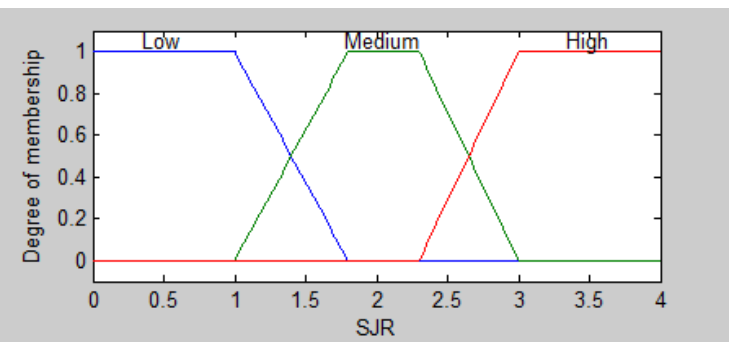

Fig. 6. Membership function for 'SJR' criterion.

The recommendation output is divided into 3 categories.

The recommendation is considered good if the output is between 0.0 and 0.25 . The recommendation is considered very good if the output is between 0.25 and 0.75 . The recommendation is considered excellent if the output is between 0.75 and 1.0. Fig. 7 shows the defuzzification phase for Recommendation output.

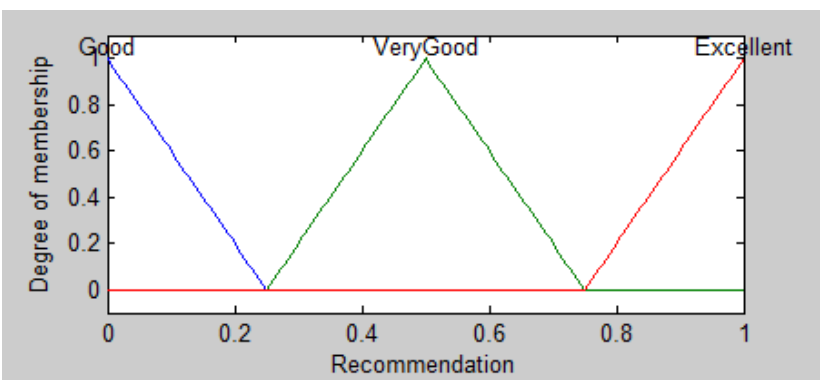

Fig. 7. Defuzzification for 'Recommendation' output.

To help users distinguish items based on their importance, color coding is used in the nodes to show the membership degrees of each node that is displayed. 20 different colors are used to present information about membership degrees as shown in Fig. 8.

In Fig. 8, 20 levels of color coding starts with color blue, followed by green, yellow and orange respectively and ends with color red. Color red is used to denote the items with the highest membership degree. The membership degree decreases as it reaches the color blue. As the membership degree ranges from 1-100, it is divided into 20 equal intervals and each interval is represented by one color in the schema. The highest membership degree which is the interval between 96 and 100 is represented with the color red, which is hottest color tone in the scale. As the membership degree decreases, corresponding color tones become closer to the color blue, which represent the least membership degree interval, which is between 1 and 5 .

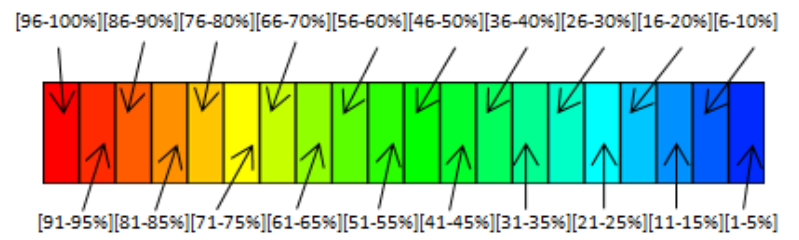

Fig. 8. Different colors used to visualize recommendation result to the users

\section{RESULTS AND DISCUSSIONS}

TABLE II: RESUlt FOR “MulTiCRITERIA DECISION” AND “SySTEM DEVELOPMENT"

\begin{tabular}{|c|c|c|c|c|c|}
\hline Domain & $\begin{array}{c}\text { Paper } \\
\text { number }\end{array}$ & SJR & Year & Citation & $\begin{array}{c}\text { Membership } \\
\text { Degrees }\end{array}$ \\
\hline \multicolumn{6}{|c|}{ Keyword : Multicriteria Decision } \\
\hline Theo. & 837709 & 0.823 & 2000 & 0 & 0.0800 \\
\hline AI & 857751 & 1.806 & 2007 & 8 & 0.3970 \\
\hline AI & 2919425 & 1.806 & 2010 & 0 & 0.0800 \\
\hline AI & 880000 & 1.506 & 2003 & 0 & 0.0889 \\
\hline AI & 857728 & 1.806 & 2007 & 0 & 0.0946 \\
\hline DB & 884509 & 1.068 & 2006 & 0 & 0.0847 \\
\hline \multicolumn{6}{|c|}{ Keyword : System Development } \\
\hline Theo. & 3161643 & null & 2010 & 17 & 0.5000 \\
\hline SE & 1075897 & 0.456 & 1999 & 0 & 0.0800 \\
\hline SE & 997582 & 0.64 & 1984 & 0 & 0.1123 \\
\hline SE & 997111 & 0.64 & 2004 & 25 & 0.5000 \\
\hline SE & 997048 & 0.64 & 1983 & 0 & 0.1173 \\
\hline SE & 997044 & 0.64 & 1992 & 0 & 0.0824 \\
\hline SE & 996559 & 0.64 & 1996 & 0 & 0.0800 \\
\hline SE & 995754 & 0.64 & 1996 & 0 & 0.0800 \\
\hline Interd. & 783282 & 0.349 & 1999 & 0 & 0.0800 \\
\hline $\mathrm{HCI}$ & 948028 & 0.334 & 2000 & 0 & 0.0800 \\
\hline $\mathrm{HCI}$ & 831661 & 0.751 & 2005 & 8 & 0.3900 \\
\hline MM & 1143179 & 0.593 & 2008 & 4 & 0.0902 \\
\hline AI & 3395084 & 0.271 & 2000 & 0 & 0.0800 \\
\hline AI & 831661 & 0.751 & 2005 & 8 & 0.3900 \\
\hline AI & 3321716 & 0.316 & 2007 & 0 & 0.0946 \\
\hline DB & 2998626 & 1.628 & 1993 & 5 & 0.0946 \\
\hline DB & 2998588 & 1.628 & 1993 & 0 & 0.0837 \\
\hline DB & 2998492 & 1.628 & 1995 & 0 & 0.0837 \\
\hline DB & 2998501 & 1.628 & 1995 & I0 & 0.0837 \\
\hline
\end{tabular}

In the experiment, several keywords have been used to find domains that are related to the keyword to be recommended. For the keyword "Multicriteria Decision", there were 6 papers found that matches the keyword. Paper \#857751 from Artificial Intelligence domain achieves the highest membership degrees, 39.7\%. While paper \#837709 from Theoretical Computer Science domain achieves the lowest score of membership degrees, 8.0\%. For the keyword "System Development", paper \#3161643 from Theoretical Computer Science domain and paper \#997111 from Software Engineering domain achieves the highest score of 
membership degrees at 50\%. Table II shows the full membership degrees score for each paper that has been found based on the supplied keyword.

Fig. 9 shows the recommendation percentage of papers that match the keyword "Multicriteria Decision". A total of 3 papers have significantly higher membership score as opposed to other papers. Fig. 10 shows the recommendation percentage of papers that matched the keyword "System Development", where only one paper has a significantly higher percentage than other papers.

Based on the membership degrees score, the result of the recommendation is shown in network visualization on the Cross Domain Recommendation Visualization System, as shown in Fig. 11. In the prototype, users will enter a keyword, select domain preference, if any, and click the Find button.

Each paper will be represented by a vertex that shows the paper number and domain. Each vertex is colored according to the paper's membership degree score. Users can select any vertex and the paper's description such as its authors, title, publication year, publication venue, and citation count will be shown. Based on this information, users can decide on their future actions.

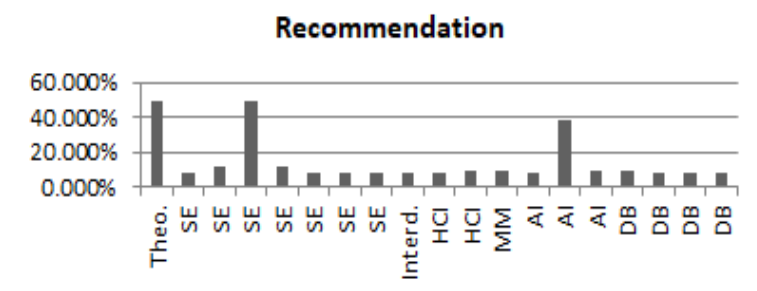

Fig. 9. Recommendation percentage for the keyword 'multicriteria decision'.

Recommendation

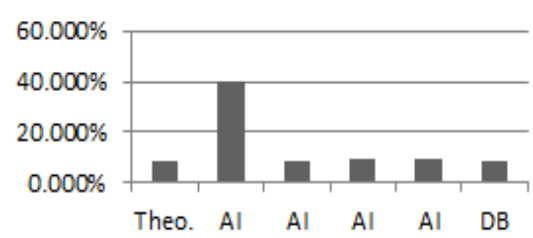

Fig. 10. Recommendation percentage for the keyword 'system development'.

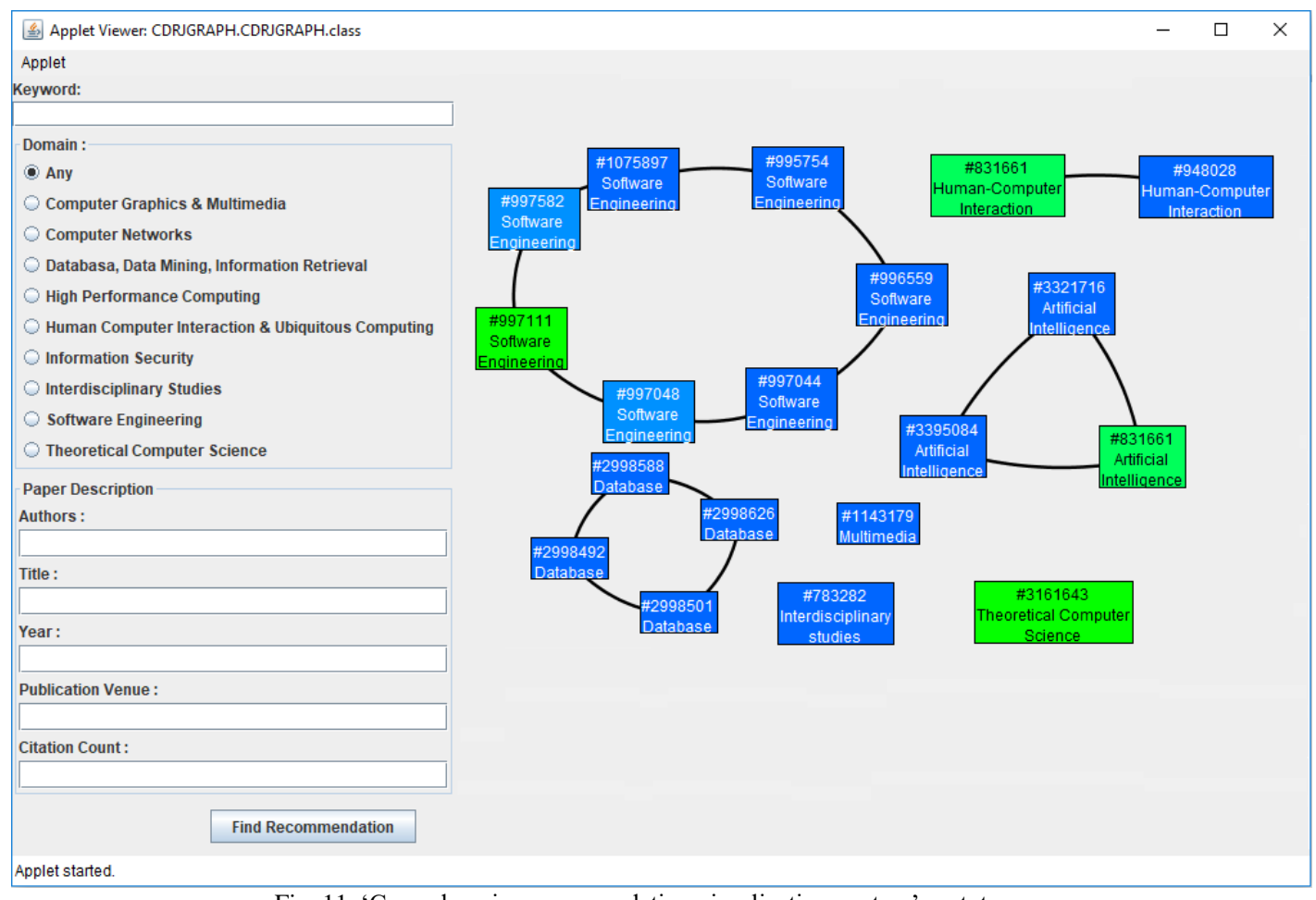

Fig. 11. 'Cross domain recommendation visualization system' prototype.

\section{CONCLUSION}

A hybrid multi-criteria decision making technique is proposed where fuzzy AHP is combined with fuzzy TOPSIS to evaluate cross domain collaboration alternatives in order to present the highly related domain with the input supplied by initial collaborator. By combining fuzzy AHP and fuzzy TOPSIS, results that are visualized to show the ranking of each related domains, researchers are able to find domains that are highly related to their interest to collaborate with. Based on the input from the researchers, the result is visualized in network form that shows each related domain. Each node represents a paper in the domain and colored according to its priority level. The visualization helps researchers to focus on the domain that are highly related to their desired criteria. When the node is selected, further information regarding the publication is displayed such as paper title, authors, publication year, and the name of the journal or conference proceedings. The hybrid multi-criteria decision making method is targeted to overcome the limitations of singular method that might overlook the accuracy of the decision making. To further improve the proposed hybrid method, the time taken to produce the visualization result should be shorter. The amount of bibliographic data is increasing rapidly each day, hence the process to find and recommend potential cross domain collaboration gets more challenging by the day, and this make it an interesting research to focus on. Researchers are still in need for big data visualization that is quick to comprehend yet give a thorough vision of what they are looking for. Users who can benefit from the proposed method 
include data scientists, business analysts, researchers, and students who are interested to form collaborations with any domains that they wish.

\section{REFERENCES}

[1] J. Tang et al., "Cross-domain collaboration recommendation," in Proc. the 18th ACM SIGKDD Intl. Conf. on Knowledge Discovery and Data Mining, 2012, pp. 1285-1293.

[2] Y. Guo and X. Chen, "A framework for cross-domain recommendation in folksonomies," J. Automation and Control Eng., vol. 1, no. 4, pp. 326-331, 2014.

[3] S. Sahebi and T. Walker, "Content-based cross-domain recommendations using segmented models," presented at the Workshop on New Trends in Content-based Recommender Systems CBRecSys@RecSys., Silicon Valley, CA, USA, October 6, 2014.

[4] A. Taneja and A. Arora, "Cross domain recommendation using multidimensional tensor factorization,” Exp. Sys. w. App., vol. 92, pp. 304-316, 2018

[5] Q. Zhang et al., "A cross-domain recommender system with consistent information transfer," Decision Support Systems, vol. 104, pp. 49-63, December 2017.

[6] Y. M. Wang and K. S. Chin, "Fuzzy analytic hierarchy process: A logarithmic fuzzy preference programming methodology," Int. J. of Approx. Reas., vol. 52, no. 4, pp. 541-553, 2017.

[7] S. Nadaban, S. Dzitac, and I. Dzitac, "Fuzzy TOPSIS: A general view," in Proc. Comp. Sci., vol. 91, pp. 823-831, 2016.

[8] M. Jakšić et al., "Fuzzy approach in ranking of banks according to financial performances," Math. Prob. Eng., p. 11, 2016.

[9] B. Pham, A. Streit, and R. Brown, Trends in Interactive Visualization, London, U.K: Springer, 2009, ch. 2, pp. 19-48.

[10] M. Zolkepli, F. Dong, and K. Hirota, "Visualizing fuzzy relationship in bibliographic big data using hybrid approach combining fuzzy c-means and newman-girvan algorithm," JACIII, vol. 18, no. 6, pp. 896-907, 2014.

[11] M. Zolkepli, F. Dong, and K. Hirota, "Automatic switching of clustering methods based on fuzzy inference in bibliographic big data retrieval system," Int. J. Fuzzy Log. Intell. Syst., vol. 14, no. 4, pp. 256-267, 2014.

[12] M. Zolkepli, F. Dong, and K. Hirota, "Visualization of fuzzy relationship using clustering algorithms in bibliographic big data," presented at 14th Intl. Symp. on Advanced Intelligent Systems (ISIS2013), Daejeon, Korea, November 13-16, 2013.

[13] M. Zolkepli, F. Dong, and K. Hirota, "Application of Fuzzy Inference Engine as an Automatic Switch between ensembles of Clustering Methods," presented at 2014 Joint 7th International Conference on Soft Computing and Intelligent Systems (SCIS) and 15th International Symposium on Advanced Intelligent Systems (ISIS), Kitakyushu, Japan, 3-6 Dec., 2014.

[14] L. A. Zadeh, "Fuzzy sets," Information and Control, vol. 8, pp. 338353,1965

[15] C. V. Negoita, Expert Systems and Fuzzy Systems, Benjamin/Cummings, Menlo Park, CA, 1985.

[16] H. J. Zimmermann, Fuzzy Sets Theory and Its Applications, Kluwer-Nijhoff, Boston-Dordrecht-Lancaster, 1985.

[17] Z. Ayag, "A fuzzy AHP-based simulation approach to concept evaluation in a NPD environment," IIE Transactions, vol. 37, pp. $827-842,2005$.

[18] F. E. Boran, S. Genç, and D. Akay, "Personnel selection based on intuitionistic fuzzy sets," Human Factors and Ergonomics in Manufacturing \& Service Industries, vol. 21, no. 5, pp. 493-503, 2011.

[19] T. L. Saaty, Analytic Hierarchy Process, New York: McGraw Hill, 1980.

[20] C. Kahraman, A. Beskese, and D. and Ruan, "Measuring flexibility of computer integrated manufacturing systems using fuzzy cash flow analysis," Information Sciences, vol. 168, pp. 77-94, 2004.
[21] O. Kilincci and S. A. Onal, "Fuzzy AHP approach for supplier selection in a washing machine company," Exp. Sys. w. App., vol. 38, no. 8, pp. 9656-9664, 2011.

[22] I. Chamodrakas, D. Batis, and D. Martakos, "Supplier selection in electronic marketplaces using satisficing and fuzzy AHP," Exp. Sys. w. App., vol. 37, pp. 490-498, 2010.

[23] O. Duran and J. Aguilo, "Computer-aided machine-tool selection based on a fuzzy-AHP approach,” Exp. Sys. W. App., vol. 34, no. 3, pp. 1787-1794, 2008.

[24] P. J. M. Van Laarhoven, and W. Pedrycz, "A fuzzy extension of Saaty's priority Theory," Fuzzy Sets Syst., vol. 11, no. 1-3, pp. 199-227, 1983.

[25] J. J. Buckley, "Fuzzy hierarchical analysis," Fuzzy Sets Syst., vol. 17, no. 3, pp. 233-247, 1983.

[26] C. L. Hwang and K. Yoon, Multiple Attributes Decision Making Methods and Applications, 1st ed., Berlin: Springer, 1981.

[27] T. Y. Chen and C. Y. Tsao, "The interval-valued fuzzy TOPSIS methods and experimental analysis," Fuzzy Sets Syst., vol. 159, no. 11, pp. 1410-1428, 2007.

[28] Z. Gligoric, C. Beljic, and V. Simeunovic, "Shaft location selection at deep multiple orebody deposit by using fuzzy TOPSIS method and network optimization," Exp. Sys. W. App., vol. 37, pp. 1408-1418, 2010.

[29] D. Yong, "Plant location selection based on fuzzy TOPSIS," Int. J. Adv. Manu. Tech., vol. 28, pp. 839-844, 2006.

[30] C. T. Chen, "Extensions of the TOPSIS for group decision-making under fuzzy environment," Fuzzy Sets Syst., vol. 114, pp. 1-9, 2000.

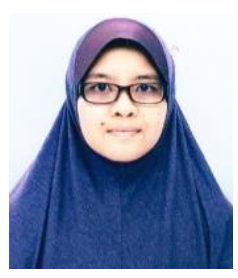

Maslina Zolkepli was born in Kuala Lumpur, Malaysia in 1983. She received her bachelor's degree in computer science in 2007, master's degree in computer science in 2010, both from Universiti Putra Malaysia, and obtained the doctor of engineering degree in computational intelligence from Tokyo Institute of Technology, Japan in 2015.

She currently is working as a senior lecturer at the Department of Computer Science and a member of the Intelligent Computing Research Group at the Faculty of Computer Science and Information Technology, Universiti Putra Malaysia. Among her recent projects are the bibliographic big data visualization approach and fuzzy analytic hierarchy process in natural disaster forecasting. She is also currently engaged in a consultation project involving the design of the fuzzy aggregation based data analytics for security threat profiling with Malaysia's cyber threat agency. Her research interests include big data, fuzzy systems, and computational intelligence.

Dr. Maslina is a member of Japan Society for Fuzzy Theory and Intelligent Informatics since 2012. She received the Monbukagakusho Scholarship Award to pursue her doctoral degree at the Tokyo Institute of Technology, Japan in 2012.

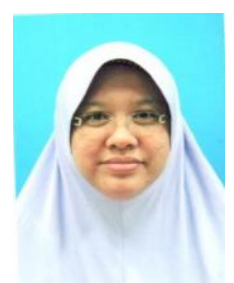

Teh Noranis Mohd Aris received her bachelor degree in information technology (Hons.) from University Utara Malaysia (UUM), in 1994. She received her master of science in artificial intelligence from University Putra Malaysia (UPM) in 2002, and Ph.D. degree from University Kebangsaan Malaysia (UKM) in 2008. Currently, she is an associate professor at the Faculty of Computer Science and Information Technology, UPM. Her research interest are Artificial Intelligence (AI), software agent and programming science. 